https://artnodes.uoc.edu

\title{
Arte y realidad aumentada. llusiones ópticas en el espacio híbrido
}

\author{
Borja Jaume Pérez \\ Universidad Complutense de Madrid
}

Fecha de presentación: octubre de 2020

Fecha de aceptación: marzo de 2021

Fecha de publicación: julio de 2021

\section{Cita recomendada}

Jaume Pérez, Borja. 2021. «Arte y realidad aumentada. Ilusiones ópticas en el espacio híbrido». Artnodes, núm. 28. UOC. [Consulta: dd/mm/aa]. http://doi.org/10.7238/a.v0i28.375418

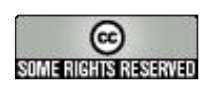

Los textos publicados en esta revista están sujetos -si no se indica lo contrario- a una licencia de Reconocimiento 4.0 Internacional de CreativeCommons. La licencia completa se puede consultar en https://creativecommons.org/licenses/by/4.0/deed.es_ES.

\section{Resumen}

Las ilusiones ópticas y la realidad aumentada (RA) utilizadas en el ámbito artístico son conceptos que han sido ampliamente estudiados por separado, pero muy pocas investigaciones han tratado el fenómeno de las ilusiones ópticas en piezas artísticas de vanguardia, creadas mediante RA. Dentro de este campo de estudio, otro factor a tener en cuenta son las variaciones a nivel perceptual de los engaños, las ambigüedades y los equívocos producidos por las ilusiones ópticas, si sus efectos se comparan entre escenarios de RA y aquellos entornos totalmente reales. Con la intención de tener un mejor entendimiento de esta cuestión, se estudian, por un lado, las obras de varios artistas, generadas a través de RA, donde se producen ilusiones ópticas; y, por el otro, se lleva a cabo un experimento en el que una serie de participantes evalúan tres piezas artísticas dinámicas, donde se superponen elementos digitales de diferentes formas, colores y dimensiones sobre fondos cuyas características generan procesos de discordancia y tensión entre figura y fondo.

\section{Palabras clave}

realidad aumentada, ilusiones ópticas, arte, percepción 


\title{
artnodes
}

https://artnodes.uoc.edu

Arte y realidad aumentada. Ilusiones ópticas en el espacio híbrido

\section{Art and augmented reality. Optical illusions in hybrid space}

\begin{abstract}
Optical illusions and augmented reality (AR) used in the artistic sphere are concepts which have been widely studied as separate, but very little has addressed the phenomenon of optical illusions in avantgarde artistic pieces created by means of AR. Within this field of study, another factor to be considered is the variations on a perceptual level of the tricks, ambiguities and misunderstandings produced by optical illusions, if their effects are compared between $A R$ settings and those environments which are completely real. With the intention of gaining a better understanding of this issue, on the one hand the works of various artists are studied which are generated by AR and where optical illusions are produced. And on the other hand, an experiment is carried out in which a series of participants evaluate three dynamic artistic pieces, in which digital elements of different forms, colours and dimensions are superimposed onto backgrounds whose characteristics generate processes of discordance and tension between outline and background.
\end{abstract}

\author{
Keywords \\ augmented reality, optical illusions, art, perception
}

\section{Introducción. La práctica artística a través de RA como fenómeno para la exploración de la percepción sensorial}

El conocimiento sobre las ilusiones provocadas por la percepción visual, independientemente del medio en el que se reproduzcan, es un campo de investigación fascinante que permite probar y verificar cualquier posible nuevo modelo de visión (Gomez-Villa, Martin, Vazquez-Corral, y Bertalmio, 2019). Además, los fenómenos ilusorios aún plantean muchas incógnitas sin resolver (Coren, y Girgus, 2020) y no parece haber un factor común único que explique las diferencias individuales en la percepción de las ilusiones ópticas (Cretenoud, Karimpur, Grzeczkowski, Francis, Hamburger, Herzog, 2019). Dentro del campo del arte se han realizado estudios sobre los engaños visuales generados a partir de ambigüedades, distorsiones, paradojas, distancia, velocidad o tamaño (Chamberlain, Drake, Kozbelt, Hickman, Siev, y Wagemans, 2017). También desde otras disciplinas, como la psicología, la neurociencia o la filosofía, se ha buscado ahondar en las causas que provocan las percepciones ilusorias (Coren, y Girgus, 2020). Algunas de las razones conocidas que pueden ocasionar estas alteraciones son las perturbaciones ópticas que intervienen entre el objeto y la retina o las señales neuronales alteradas (Gomez-Villa, Martin, Vazquez-Corral, Bertalmio, 2019). Estos desajustes entre realidad y percepción proporcionan conocimientos que pueden ser muy útiles para desarrollar nuevos modelos de visión de la percepción y la cognición.

A pesar de la gran cantidad de investigaciones sobre RA realizadas en los últimos años, como consecuencia del importante desarrollo de los dispositivos que soportan esta tecnología, se han realizado pocos estudios dedicados a identificar aspectos relacionados con las ilusiones ópticas al combinar entornos reales y virtuales, más allá de las ilusiones visuales provocadas por la estimación de las distancias (Lin, Caesaron, Woldegiorgis, 2019). Teniendo en cuenta las posibilidades que ofrece la RA, es necesario abordar el estudio de las relaciones perceptivas resultantes, no solo a través de campos como la ingeniería informática, la neurociencia o la psicología, sino también desde un punto de vista artístico, ya que la fenomenología visual es una de las bases de la investigación, no solo en ciencia, sino también en arte.

Este artículo presenta un estudio sobre las posibles alteraciones derivadas de las ilusiones ópticas en obras artísticas de RA, teniendo en cuenta los principios de la visión binocular y la dinámica humana, que produce la percepción de profundidad y movimiento (Hibbard, Haines, y Hornsey, 2017).

El trabajo posterior se estructura de la siguiente manera: sección 2 , se describen investigaciones, antecedentes históricos y trabajos artísticos relevantes relacionados con las ilusiones ópticas y la percepción visual en entornos reales y aumentados; sección 3, se muestran una serie de prototipos artísticos realizados por el autor y se hace una descripción en función del tamaño, distancia entre figura y fondo, color y forma; sección 4, se discuten los resultados obtenidos a partir de las pruebas realizadas a varios usuarios; sección 5 , se presentan conclusiones e investigaciones futuras.

\section{Trabajo relacionado}

Las ilusiones ópticas se pueden clasificar en dos grupos principales: fisiológicas y cognitivas (Díaz Padilla, 2010). Si bien, existen otros entornos y medios donde conviene analizar su comportamiento, como 


\section{artnodes}

https://artnodes.uoc.edu

es el caso de la RA, basada en la inclusión de capas de contenido virtual sobre el entorno real mediante el uso de algún dispositivo (smartphone, visor RA) (Slater, Gonzalez-Liencres, Haggard, Vinkers, Gregory-Clarke, Jelley, Watson, Breen, Schwarz, Steptoe, Szostak, Halan, Fox, y Silver, 2020).

\subsection{Ilusiones ópticas: teorías y procesos para la práctica artística}

En el campo perceptivo, las ilusiones ópticas son las alteraciones que se producen cuando hay una discrepancia entre la percepción de un elemento 0 imagen y sus características originales en el entorno físico (Díaz Padilla, 2010). Desde la antigüedad se han observado las divergencias entre percepción y realidad, Platón, con su alegoría de la caverna, es un ejemplo. Aristóteles fue quien dio a conocer la ilusión en la que, al mirar una cascada durante un largo período, los elementos circundantes parecían moverse en dirección opuesta (Bach, y Poloschek, 2006). Los artistas están capacitados para conocer los efectos que provoca la disposición de los elementos de una u otra manera y han utilizado todo tipo de trucos para representarlos en sus obras (Díaz Padilla, 2010). En el siglo xx, el Op Art (Optical Art) apareció como un género artístico donde la ciencia y el arte se mezclaban; una variante de abstracción que generaba una impresión visual sorprendente mediante el uso de propiedades dinámicas como la perspectiva o la tensión cromática (Huang, 2020). Las pinturas de la artista Bridget Riley son un claro exponente del uso artístico de los mecanismos de la percepción (Dodgson, 2008; Zanker, y Walker, 2004; Zeki, y Lamb, 1994). Rycroft afirmaba que estas pinturas ejercían una activación de la conciencia cognitiva debido a la experiencia perceptiva del movimiento (Rycroft, 2005). Exposiciones recientes, como Victor Vasarely The birth of Op Art, Museo Thyssen-Bornemisza (2018), u Op Art in focus, Tate Museum of Modern and Contemporary British Art (2020), demuestran un interés permanente en este género.

El creciente desarrollo de los ordenadores, los dispositivos móviles y la aparición de la RA ha permitido a los artistas desarrollar nuevos discursos y explorar las posibilidades preceptivas en el entorno aumentado (Rhodes, 2018). Wade señala que las imágenes planas pueden presentar paradojas perceptivas cuando se presentan a través de pantallas (2011). Otros estudios plantearon interrogantes sobre la distinción perceptual que se debe hacer entre «analógico» y «digital» (Gomez-Villa, Martín, Vazquez-Corral, Malo, y Bertalmío, 2019), o los factores que influyen en la captura de imágenes en dispositivos digitales, como la resolución y el contraste del dispositivo de visualización, el nivel y rango de luminancia o la relación señal/ ruido de la imagen visualizada (Nather, Mecca, Bueno, 2012). Otros estudios destacan la importancia del campo de visión o las propiedades y la fidelidad del color de los dispositivos (Gruber, Kalkofen, y Schmalstieg, 2010). Todo esto genera distintas distorsiones que afectan al escenario donde se reproduce la RA, lo cual supone un nuevo campo de exploración para los artistas, permitiéndoles a su vez aportar sus singulares capacidades cognitivas de interpretación
Arte y realidad aumentada. Ilusiones ópticas en el espacio híbrido

(Chamberlain, Drake, Kozbelt, Hickman, Siev, y Wagemans, 2017) en relación con la percepción del espacio circundante.

\subsection{Color y percepción: rasgos fundamentales y piezas artísticas de RA}

Tamiko Thiel (2018) es una artista digital que utiliza la RA de manera recurrente en su obra. Un ejemplo es la pieza Evolution of Fish(2019) (fig. 1), donde reflexiona sobre la amenaza que representa para los ecosistemas el vertido de desechos plásticos en el océano. En este caso, se puede ver un engaño visual conocido como efecto espacial de los colores (Cuevas, 2010). Al colocar ciertos colores en una superficie dada, parecen estar a diferentes distancias del observador en función de su tono. Los colores con longitudes de onda cortas (azul, violeta-verde) tenderán a aparecer más lejanos, mientras que los colores con longitudes de onda largas (amarillo, naranja y rojo) se percibirán más cercanos. Esto, unido al problema de la subestimación de la profundidad en entornos virtuales (Peillard, Argelaguet, Normand, Lécuyer, y Moreau, 2019), donde se tiende a percibir erróneamente las distancias de los elementos aumentados, provoca una serie de engaños perceptivos que acentúan la ilusión.

En la obra, se pueden ver dos cardúmenes diferenciados por el color, unos generados a partir de tonos fríos (azul) y otros cálidos (rojo y naranja). La sensación es que los peces con colores cálidos parecen estar más cerca que los creados a partir de tonos fríos, cuando, en realidad, están a la misma distancia.

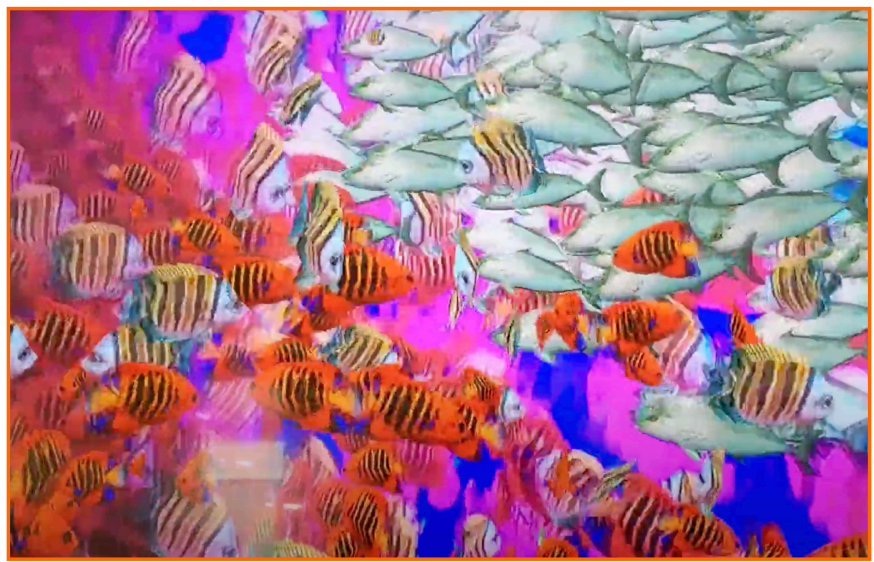

Figura 1. Evolution of Fish. Tamiko Thiel. 2019

\subsection{Ilusiones cognitivas: distorsión en piezas artísticas de RA}

Un tipo de ilusión óptica generada por la distorsión de la forma es la figura imposible. Este engaño visual se da cuando los contornos de una forma determinada se construyen en un sentido ilógico y/0 alterado (Penrose, y Penrose, 1958). Esto se hace visible en la obra de arte Large reflector \# 1 del artista Will Pappenheimer (2006) (fig. 2). En este caso, creó una escultura virtual ubicada en Central Park, Nueva York, utilizando paneles de espejos giratorios que en conjunto 


\section{artnodes}

https://artnodes.uoc.edu

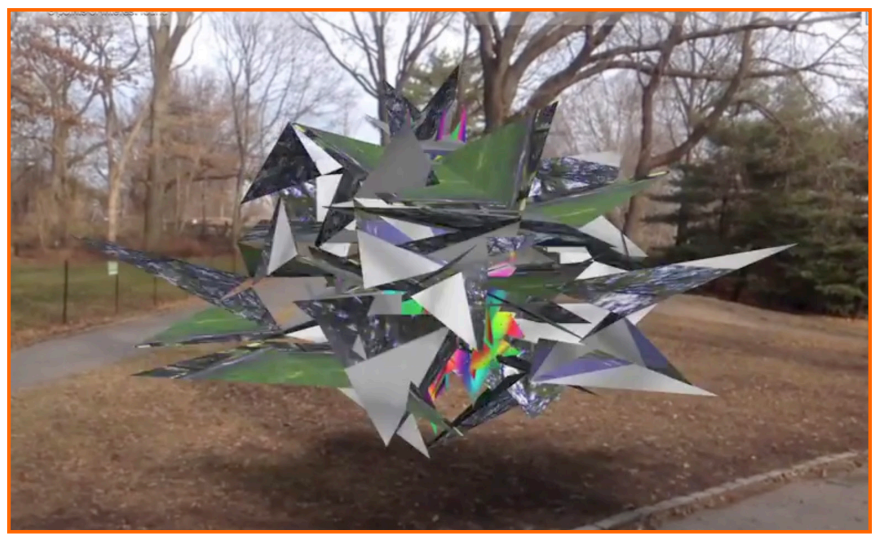

Figura 2. Large reflector \# 1. Will Pappenheimer. 2013

generan una figura imposible con una vista 360 de dicha ubicación en Google Street View.

Otro ejemplo de ilusión cognitiva es la distorsión anamórfica, basada en modelos de representación a través de la perspectiva en la construcción de figuras para lograr el engaño visual. La artista Dunaway Smith (2020) usó esta técnica para crear su obra AR Stardust (fig. 3). Cuando el usuario está frente a la obra, observa una espiral que se mueve sobre su eje central, simulando así la forma de una galaxia. Si gira hasta tener una vista lateral de la pieza, verá una serie de círculos separados de diferentes colores.

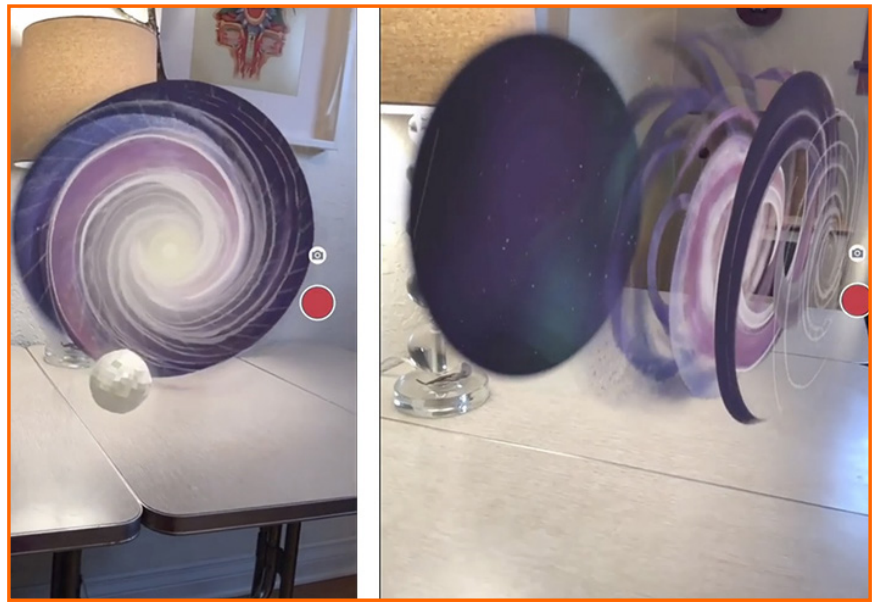

Figura 3. AR Stardust. Dunaway Smith. 2020

\subsection{El trompe L'oeil virtual: trampantojo aumentado}

Dentro de las ilusiones cognitivas se encuentra el trampantojo, un tipo de ilusión óptica con la que se pretende engañar al ojo empleando técnicas de perspectiva, siendo una técnica utilizada de manera recurrente por los artistas a lo largo de la historia.

Julian Oliver (2007) diseñó la pieza de RA Level Head en 2007 (fig. 4), en la que un personaje se mueve por diferentes habitaciones.
La mecánica del juego consiste en dirigir un dispositivo (smartphone, tablet, etc.) hacia un pequeño cubo de plástico en el que, a través de $\mathrm{RA}$, se muestra una habitación en cada una de sus caras. Dentro de la habitación, hay un personaje que es movido por el usuario, inclinando el cubo hacia un lado y el otro hasta encontrar una puerta que lleve al resto de espacios. Esta obra artística se inspira en la genealogía de los procesos de la memoria, entendida como arte, y la fragilidad de la percepción. Se pone aquí de manifiesto la técnica del trampantojo para perpetrar el engaño visual mediante el efecto de profundidad en el plano bidimensional.

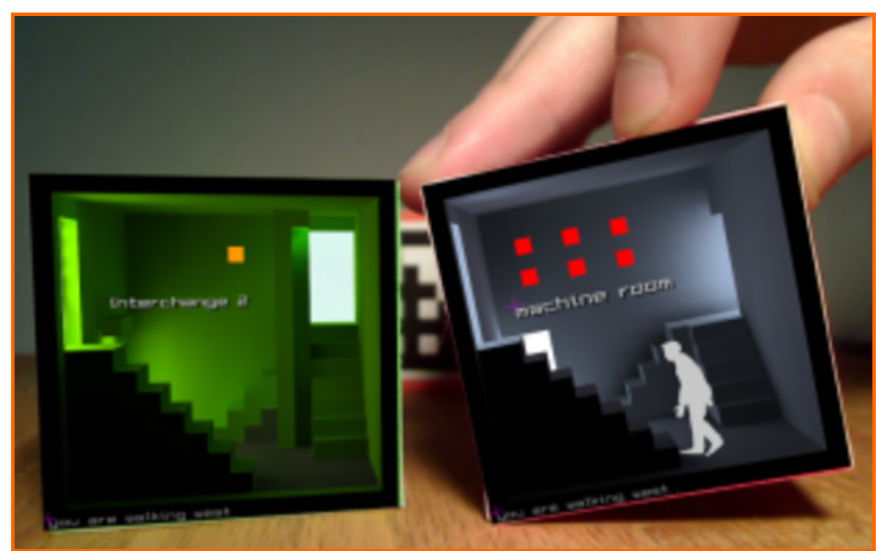

Figura 4. Level Head. Julian Oliver. 2007

\section{Ensayo práctico: diseño de tres piezas con RA}

En esta sección se presentan las piezas de RA creadas por el autor de este artículo. Se han elegido tres prototipos basados en tres tipos de ilusiones ópticas: percepción errónea del movimiento, asimilación remota de color y anamorfosis. Estas fueron elegidas porque reúnen condiciones de interés en entornos de RA, como el tamaño, la distancia entre figura y fondo, el color y la forma. Dichas piezas fueron especialmente diseñadas para esta investigación a lo largo del año 2020, utilizando formas y colores que fueran especialmente sensibles a la generación de ilusiones ópticas. Para ello, varios participantes evaluaron las ilusiones ópticas generadas a través de dispositivos monoculares (en este caso, teléfonos móviles), obviando otros sistemas de RA como las pantallas binoculares inmersivas (Head Mounted Display). A continuación, se detalla el diseño experimental y las piezas artísticas.

\subsection{Participantes}

Para el análisis de las piezas, participaron veinte voluntarios con edades comprendidas entre 26 y 58 años de edad. Todos tenían visión correcta y 12 de 20 habían utilizado previamente RA a través de alguna aplicación de teléfono inteligente. 


\section{artnodes}

https://artnodes.uoc.edu

\subsection{Proceso}

La prueba se realizó a través de la aplicación Artivive (Ardelean, y Popescu, 2017), una herramienta online que permite cargar ilustraciones bidimensionales en su plataforma e integrar elementos por capas superpuestas, que luego pueden ser visualizadas a través de RA descargando la aplicación en un smartphone. Para realizar la prueba, se colocaron las tres imágenes en una zona bien iluminada, pero sin recibir luz directa para evitar reflejos o destellos. Posteriormente los participantes debían ubicarse a distancias específicas de la obra (100 $\mathrm{cm}$ y $25 \mathrm{~cm}$ ), observarlas durante un tiempo determinado ( 20 segundos) y dirigir el dispositivo (smartphone) hacia el marcador, para ver aparecer la RA. Tras observar todas las piezas, debían completar un formulario donde se les hacía una serie de preguntas sobre sus experiencias con cada una de las piezas. Las preguntas estaban relacionadas con las sensaciones perceptivas que habían tenido, no solo a diferentes distancias, sino también desde diferentes ángulos de visión.

Las dos distancias sugeridas entre el participante y la obra (100 cm y $25 \mathrm{~cm}$ ) se eligieron según el llamado campo cercano del espacio perceptivo (Singh, Ellis, y Swan, 2020), donde la percepción de profundidad actúa con mayor precisión. La primera distancia (1 m) se eligió después de varias pruebas, que determinaron que era el punto óptimo, dentro del campo cercano, desde donde ver la pieza en su conjunto y observar correctamente las ilusiones ópticas.

\subsection{Primera pieza artística: Rotsnake aumentado. Modelo basado} en la figura Rotsnake, de Akiyoshi Kitaoka

Para este primer trabajo, inspirado en las Serpientes giratorias de Akiyoshi Kitaoka (2003), se realizó una ilustración digital con unas

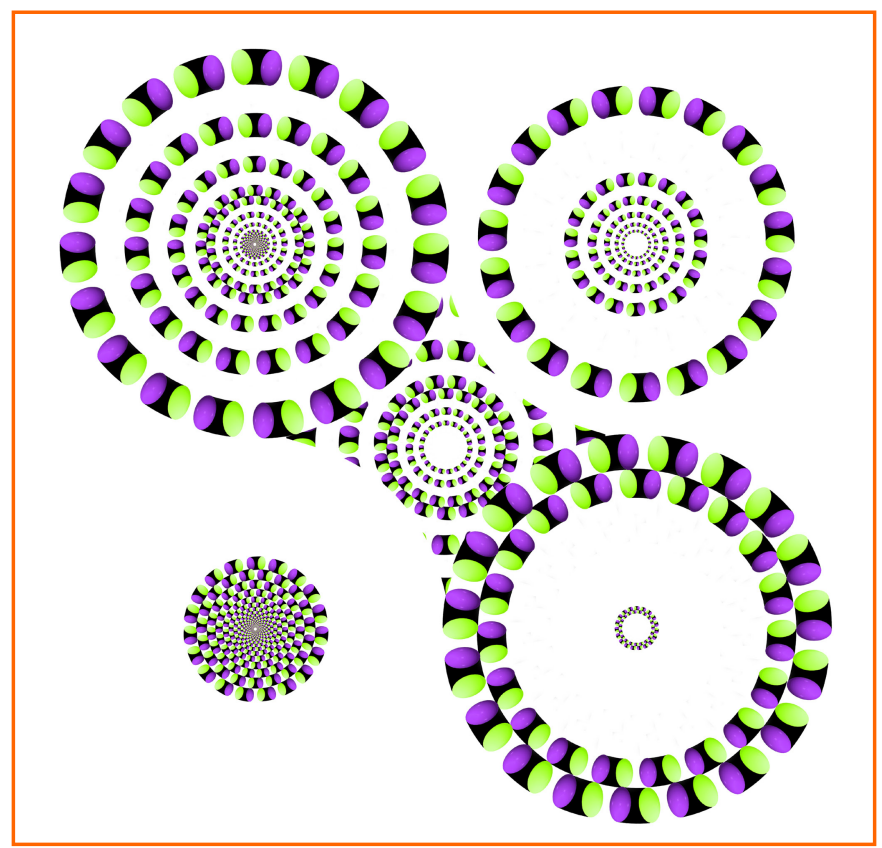

Figura 5. Modelo basado en Rotsnake, de Akiyoshi Kitaoka. Borja Jaume. 2020 dimensiones de $20 \times 20 \mathrm{~cm}$. Teniendo en cuenta las investigaciones de Akiyoshi Kitaoka, por las que los movimientos ilusorios ocurren basados en una secuencia acromática (gris oscuro-negro-gris claroblanco), posteriormente reemplazada por colores (Conway, Kitaoka, Yazdanbakhsh, Pack, y Livingstone, 2005), se realizó una versión con la siguiente secuencia cromática (violeta-negro-verde-blanco). Los participantes debían observar una imagen bidimensional compuesta por cuatro elementos con forma de engranajes (fig. 5). Para completar la pieza se debía dirigir el smartphone hacia la ilustración, generando una capa de contenido digital en RA que permitía «rellenar» los huecos de los engranajes (fig. 6 y 7). Se tuvieron en cuenta dos tipos de ilusiones ópticas: el movimiento aparente, en el que los elementos

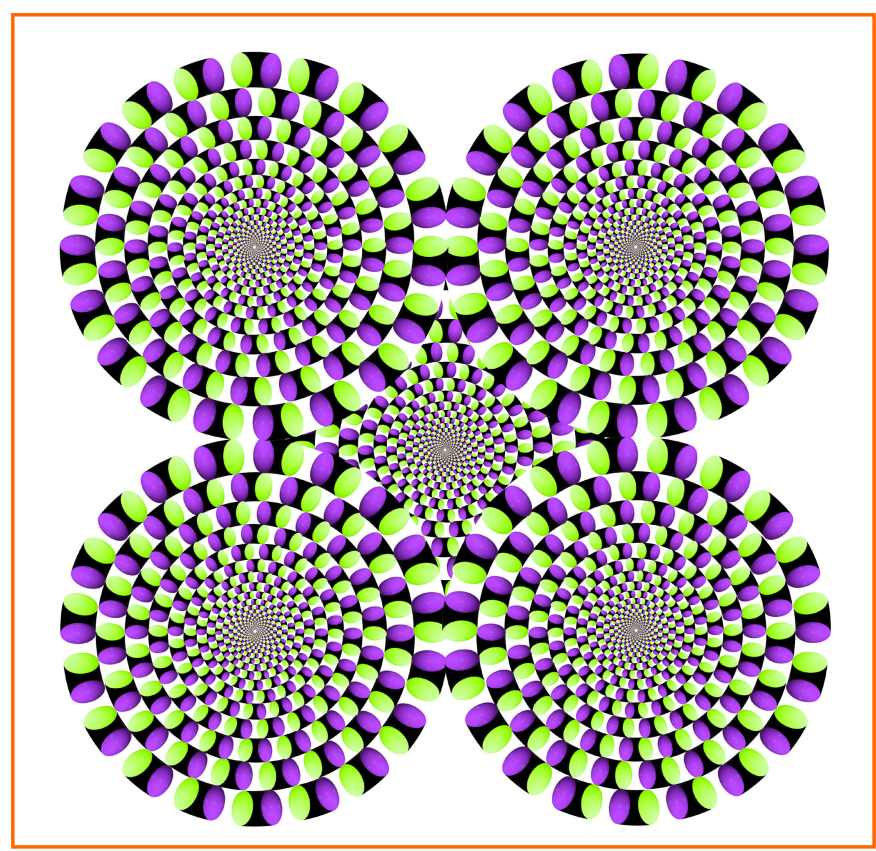

Figura 6. Modelo basado en Rotsnake, de Akiyoshi Kitaoka. Borja Jaume. 2020

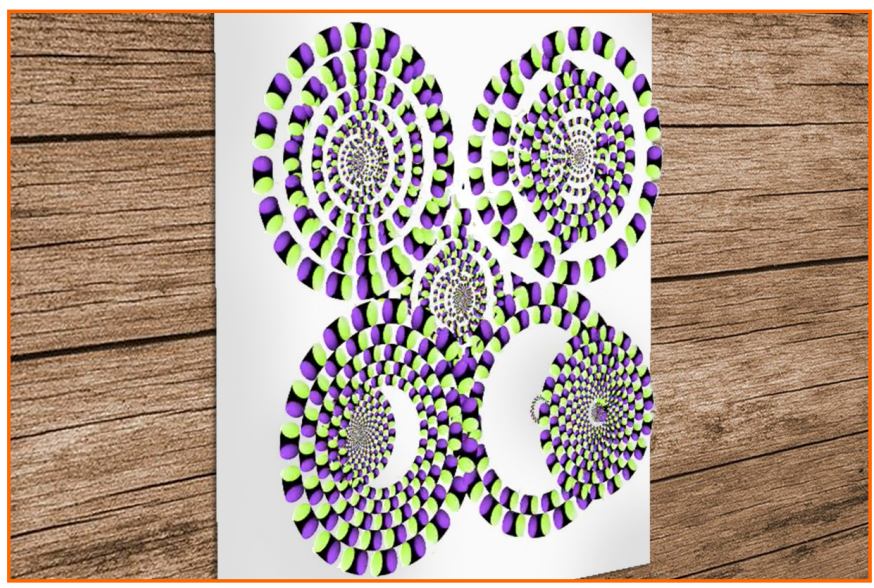

Figura 7. Modelo basado en Rotsnake, de Akiyoshi Kitaoka. Borja Jaume. 2020 


\section{artnodes}

https://artnodes.uoc.edu

se mueven a pesar de que la imagen sea estática, y la anamorfosis, que requiere estar en un determinado punto de vista para visualizar correctamente la imagen.

\subsection{Segunda pieza artística: percepción errónea del color a través de la influencia contaminante de un tercero}

Para la segunda pieza se realizó una impresión digital abstracta $(20 \mathrm{x}$ $20 \mathrm{~cm}$ ) con un fondo de color azul, rodeado en los bordes por figuras geométricas blancas y negras (fig. 8). Al igual que en la pieza Rotsnake,

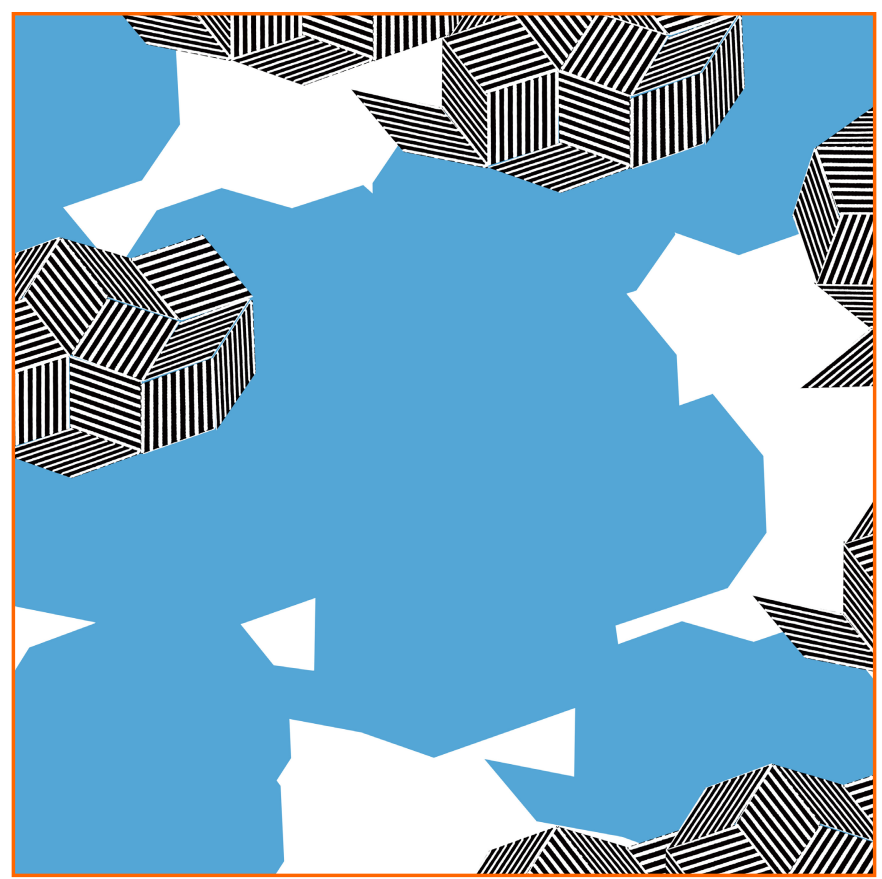

Figura 8. Grid. Borja Jaume. 2020

los participantes debían dirigir sus smartphones hacia la ilustración para completarla. La RA generada consistía en dos rectángulos superpuestos conformados por elementos geométricos de color rojo y azul, con zonas transparentes para ver el fondo (fig. 9 y 10). Para esta pieza se tuvo en cuenta la asimilación remota, por lo que los estímulos generados por los colores vecinos en un tercero, presentados simultáneamente, provocan su percepción errónea (Bach, Poloschek, 2006).

\subsection{Tercera pieza artística: Meta-morphosis. Distorsión anamórfica a través de RA}

Para la tercera pieza realizada en esta investigación se tuvo en cuenta la distorsión anamórfica en la que, para lograr una correcta representación de una determinada imagen, el espectador debe visualizarla desde un punto de vista concreto.

La obra es una impresión digital en blanco y negro, que simula un papel seccionado de tal forma que solo permite ver parte de
Arte y realidad aumentada. Ilusiones ópticas en el espacio híbrido

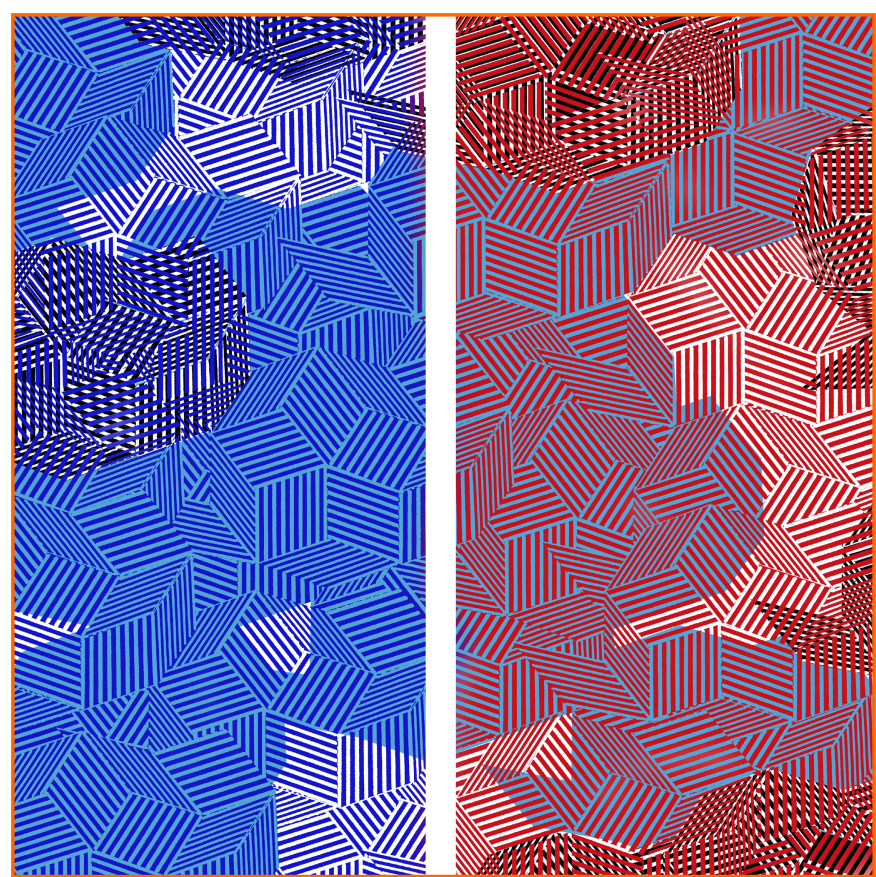

Figura 9. Grid. Borja Jaume. 2020

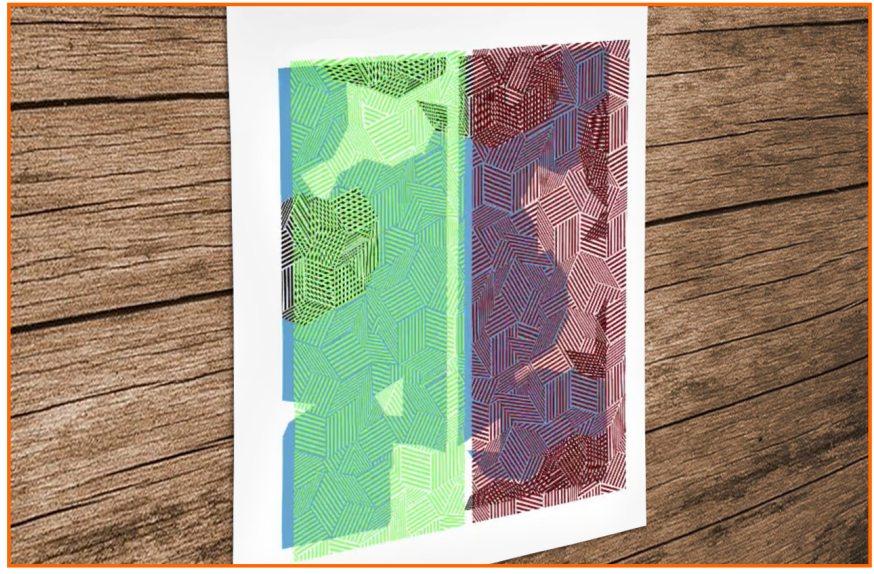

Figura 10. Grid. Borja Jaume. 2020

una figura imposible representada en el centro de la imagen (fig. 11). Dirigiendo el dispositivo móvil hacia la pieza, con la aplicación Artivive abierta, esta se "completa», permitiendo ver nuevos elementos de la ilustración (fig. 12). Además, aparecen una serie de formas suspendidas en el aire que, a través del correcto punto de vista, darán forma a otras figuras (un círculo y una roca) (fig. 13). Dichos elementos, representados a través de RA, fueron colocados a diferentes distancias y profundidades, de tal manera que, para encajar correctamente, fue necesario colocarlas a una distancia aproximada de $70 \mathrm{~cm}$, con un punto de vista lateral. 


\section{artnodes}

https://artnodes.uoc.edu

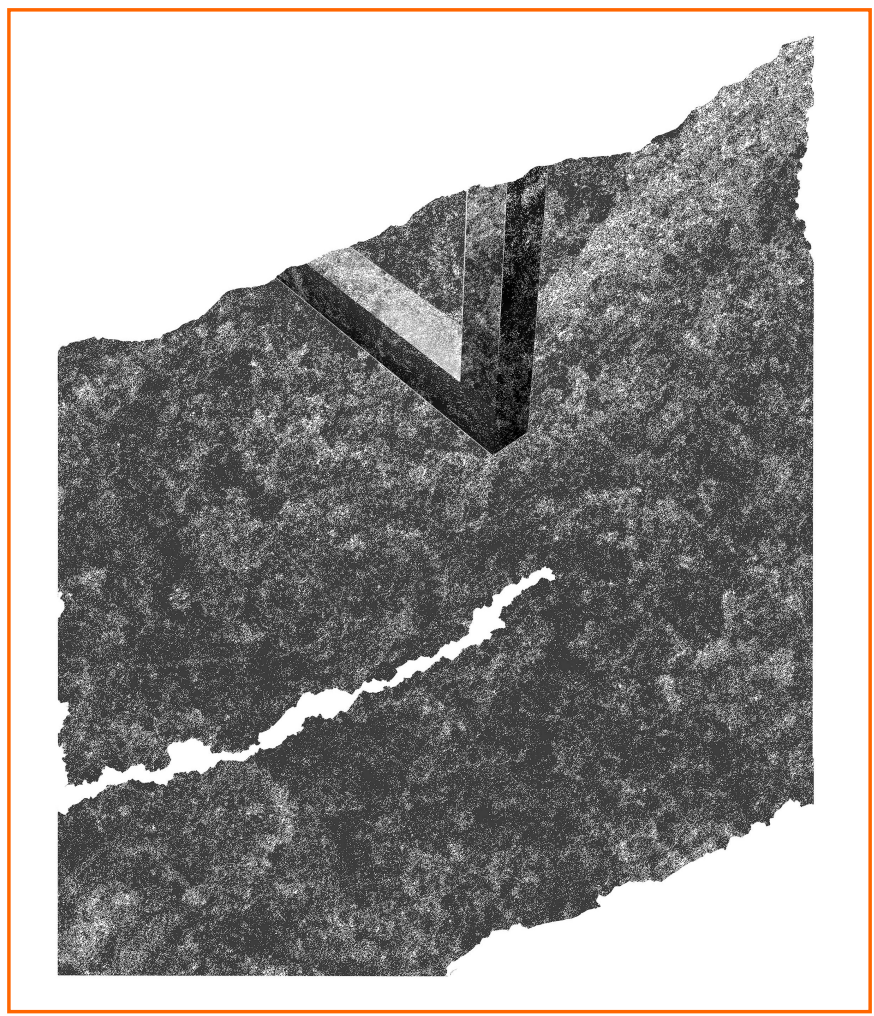

Figura 11. Metamorphosis. Borja Jaume. 2020

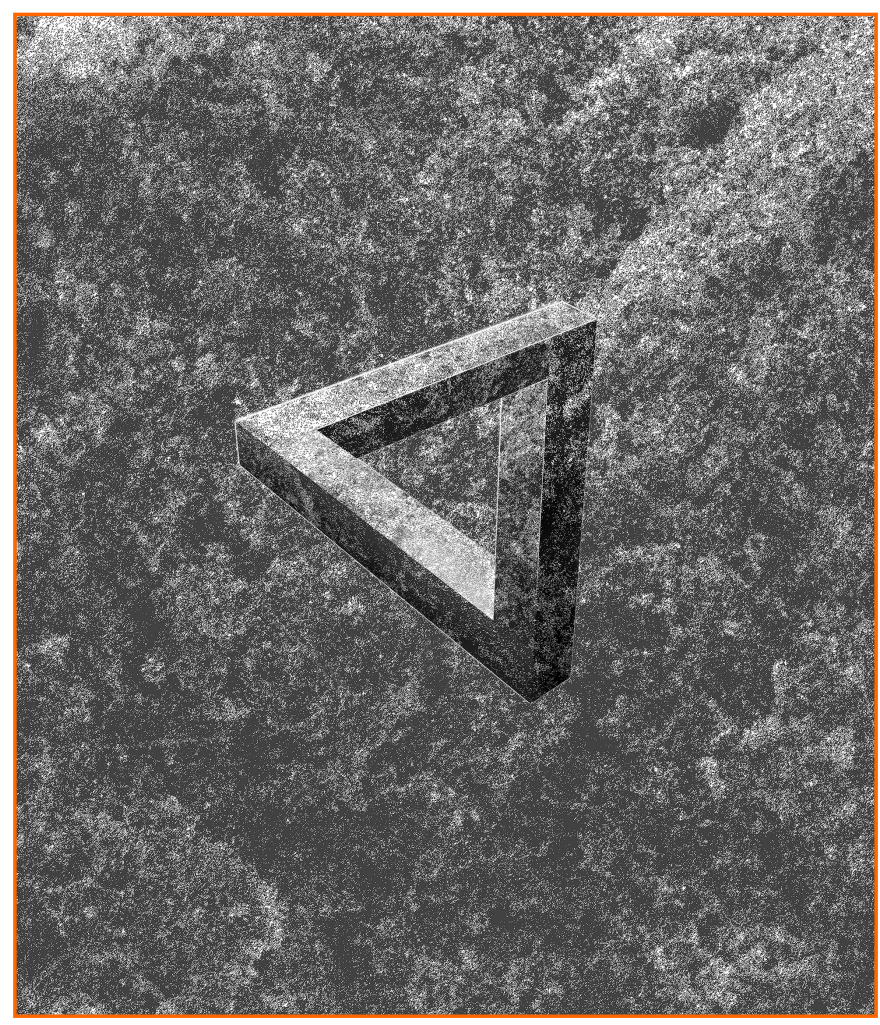

Figura 12. Metamorphosis. Borja Jaume. 2020
Arte y realidad aumentada. llusiones ópticas en el espacio híbrido

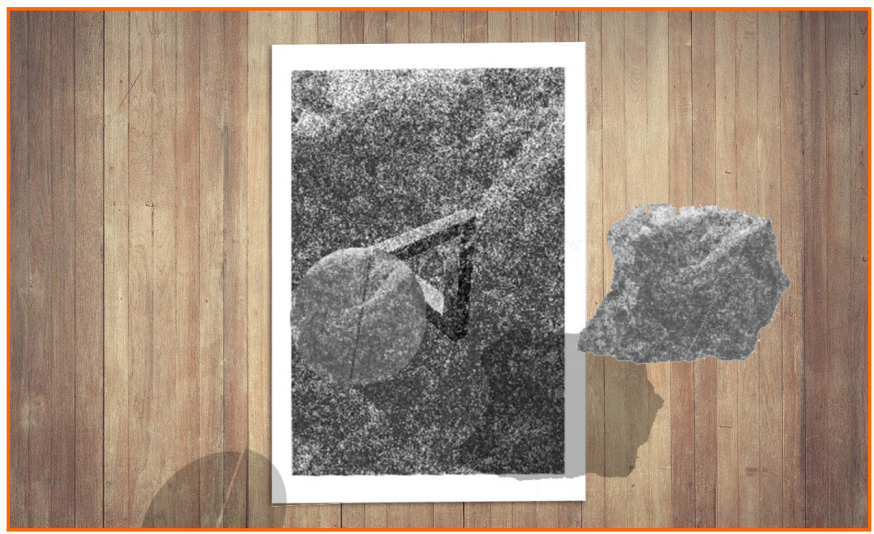

Figura 13. Metamorphosis. Borja Jaume. 2020

\section{Resultados obtenidos}

Una vez recopiladas las respuestas proporcionadas por los participantes, se obtuvieron una serie de resultados que pueden observarse en la siguiente tabla (tabla 1):

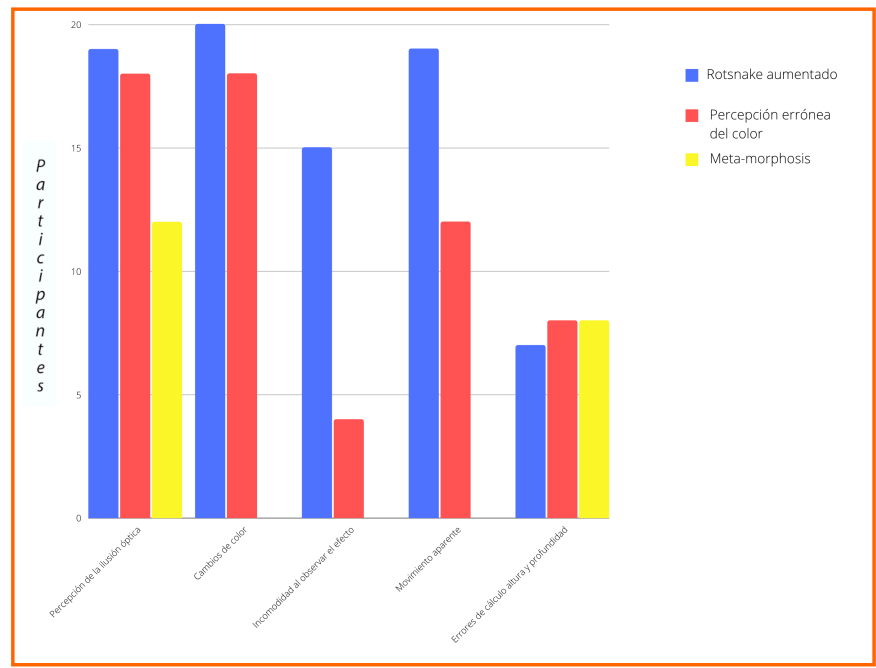

Tabla 1. Gráfico de resultados

Los participantes obtuvieron resultados similares en cuanto a la percepción del engaño óptico, siendo algo menor en la pieza Metamorphosis, debido a que varios no encontraron la conexión entre piezas. En cuanto a los cambios de color, prácticamente todos los participantes los detectaron, excepto en la tercera pieza, debido a la ausencia de color en ella. Además, en las dos primeras se produjo una notable percepción de movimiento aparente. Por último, en las tres piezas analizadas hubo un porcentaje muy similar en cuanto a errores perceptivos de distancia y altura del contenido digital respecto a la imagen analógica. 


\section{artnodes}

https://artnodes.uoc.edu

\subsection{Primera pieza artística: Rotsnake aumentado. Modelo basado en la figura Rotsnake, de Akiyoshi Kitaoka}

Diecinueve participantes detectaron movimiento aparente en la representación $\mathrm{RA}$, aunque el efecto fue menos pronunciado que cuando se observaba solo la imagen impresa (los engranajes sin RA). Un participante no detectó movimiento al verlo a través de RA.

Todos los participantes indicaron que la imagen impresa pierde intensidad de color cuando se ve a través de la pantalla, pero no así con el contenido digital en RA.

Seis participantes encontraron bastante molesto mirar la imagen, mostrando fatiga visual y mareos. Nueve participantes sintieron algo de malestar y otros cinco no tenían ningún malestar al mirarlo.

Al alejarse, dieciocho de los participantes indicaron que las ruedas seguían moviéndose, pero de forma menos acusada. Un participante indicó que las ruedas dejaron de moverse.

A siete participantes les resultó complicado definir la profundidad y la distancia del contenido RA.

\subsection{Segunda pieza artística: percepción errónea de un color a través de la influencia contaminante de un tercero}

Dieciocho participantes indicaron que el color azul del fondo cambiaba de tonalidad al superponer los cuadros con mallas de colores. Observaron un tono más claro de azul en el lado izquierdo y un tono más oscuro en el derecho. Dos participantes informaron que no hubo cambio de color.

Doce participantes indicaron que cuando se acercaron se comprobó que el fondo cambió de color, aunque no notaron un cambio mayor. Seis participantes dijeron que el cambio de color fue más pronunciado. Un participante señaló que, al acercarse, sí vio un cambio de tono que no había visto antes.

Cinco participantes indicaron ver colores inexistentes en la pieza (violeta, azul verdoso, rojo violeta) como resultado de la mezcla de los colores de la capa de RA con el fondo.

Tres participantes manifestaron que les resultó algo incómodo mirar la pieza. Un participante afirmó que le causaba fatiga visual.

Doce participantes indicaron haber percibido cierto movimiento aparente producto de la interacción del color. Además, a ocho participantes les resultó complicado definir la distancia del contenido RA.

\subsection{Tercera pieza artística: Meta-morphosis. Distorsión anamórfica a través de RA}

Siete participantes encontraron fácil unir las piezas de RA. Tres participantes tuvieron que moverse varias veces para encontrar la conexión entre piezas.

Ocho participantes informaron haber tenido problemas para identificar la posición correcta porque no tenían clara la posición y la distancia de los elementos en la RA.

Ningún participante indicó haber percibido cambios de color, incomodidad al observar la pieza o movimiento aparente.
Arte y realidad aumentada. Ilusiones ópticas en el espacio híbrido

Doce participantes consideraron que había poca distancia de profundidad en las piezas cuando estas se unían. Ocho participantes consideraron que no había distancia de profundidad entre las piezas cuando estas se unían.

\subsection{Discusión general basada en los resultados obtenidos}

En las siguientes tablas se pueden ver los resultados del estudio (tablas 2 y 3 ).

\begin{tabular}{|c|c|c|c|c|}
\hline \multirow[t]{3}{*}{ ROTSNAKE } & \multirow{2}{*}{\multicolumn{2}{|c|}{1 metro }} & \multirow{2}{*}{\multicolumn{2}{|c|}{$25 \mathrm{~cm}$}} \\
\hline & & & & \\
\hline & RA & ENTORNO FíSICO & RA & ENTORNO FÍSICO \\
\hline $\begin{array}{l}\text { Percepción de la } \\
\text { ilusión óptica }\end{array}$ & Menos pronunciado & Más pronunciado & \begin{tabular}{|l|} 
Menons pronunciado (aunque \\
mejor que a un metro)
\end{tabular} & Más pronunciado \\
\hline Cambios de color & Pérdida de intensidad & & Pérdida de intensidad & \\
\hline $\begin{array}{l}\text { Incomodidad al } \\
\text { observar la a } \\
\text { ilusión óptica }\end{array}$ & Menos pronunciado & Más pronunciado & $\begin{array}{l}\text { Menos pronunciado } \\
\text { (menos incómodo } \\
\text { que a un metro) }\end{array}$ & Más pronunciado \\
\hline $\begin{array}{l}\text { Movimiento } \\
\text { aparente }\end{array}$ & Menos pronunciado & Movimiento percibido & $\begin{array}{l}\text { Menos pronunciado } \\
\text { (mejor que a } \\
\text { un metro) }\end{array}$ & Más pronunciado \\
\hline $\begin{array}{l}\text { Alturay } \\
\text { profundidad } \\
\text { en el espacio }\end{array}$ & Identificación sencilla & & Identificación sencilla & \\
\hline \multicolumn{5}{|l|}{ COLOR MISTAKE } \\
\hline & \multicolumn{2}{|l|}{\begin{tabular}{|l|l} 
metro \\
$\mathrm{RA}$
\end{tabular}} & \multicolumn{2}{|l|}{$25 \mathrm{~cm}$} \\
\hline & RA & ENTORNO FíSICO & $\mathrm{RA}$ & ENTORNO FÍSICO \\
\hline $\begin{array}{l}\text { Percepción de la } \\
\text { ilusión óptica }\end{array}$ & Percepción correcta & Percepción correcta & Percepción correcta & Percepción correcta \\
\hline Cambios de color & $\begin{array}{l}\text { Percepción de colores } \\
\text { inexistentes }\end{array}$ & $\begin{array}{l}\text { Percepción errónea } \\
\text { del color }\end{array}$ & $\begin{array}{l}\text { Percepción errónea } \\
\text { del color }\end{array}$ & $\begin{array}{l}\text { Percepción errónea } \\
\text { del color }\end{array}$ \\
\hline $\begin{array}{l}\text { Incomodidadal abservar } \\
\text { la lilusion oppica }\end{array}$ & $\begin{array}{l}\text { Observación no } \\
\text { incómoda en general }\end{array}$ & $\begin{array}{l}\text { Percepción errónea } \\
\text { del color }\end{array}$ & $\begin{array}{l}\text { Observación no } \\
\text { incómoda en general }\end{array}$ & $\begin{array}{l}\text { Observación no } \\
\text { incómoda en general }\end{array}$ \\
\hline \multicolumn{5}{|l|}{$\begin{array}{l}\begin{array}{l}\text { Movimiento } \\
\text { aparente }\end{array} \\
\end{array}$} \\
\hline $\begin{array}{l}\text { Altura y } \\
\text { profundidad } \\
\text { en el espacio } \\
\end{array}$ & Identificación sencilla & & Identificación sencilla & \\
\hline \multicolumn{5}{|l|}{ METAMORPHOSIS } \\
\hline & \multicolumn{2}{|l|}{1 metro } & \multicolumn{2}{|l|}{$25 \mathrm{~cm}$} \\
\hline & RA & ENTORNO FÍSICO & $\mathrm{RA}$ & ENTORNO FÍSICO \\
\hline $\begin{array}{l}\text { Percepción de la } \\
\text { ilusión óptica }\end{array}$ & Identificación sencilla & & Identificación sencilla & \\
\hline Cambios de color & & & & \\
\hline $\begin{array}{l}\text { Incomodidad al observar } \\
\text { la ilusion optica } \\
\end{array}$ & $\begin{array}{l}\text { Observación no } \\
\text { incómoda en general }\end{array}$ & & \begin{tabular}{|l|l|} 
Observación no \\
incómoda en general
\end{tabular} & \\
\hline $\begin{array}{l}\text { Movimiento } \\
\text { aparente }\end{array}$ & & & & \\
\hline $\begin{array}{l}\text { Altura y } \\
\text { profundidad } \\
\text { en el espacio }\end{array}$ & $\begin{array}{l}\text { Errores de apreción en } \\
\text { la distancia exocéntrica }\end{array}$ & & $\begin{array}{l}\text { Errores de apreción en } \\
\text { la distancia exocéntrica } \\
\text { (Menos pronunciados) }\end{array}$ & \\
\hline
\end{tabular}

Tabla 2. Resultados

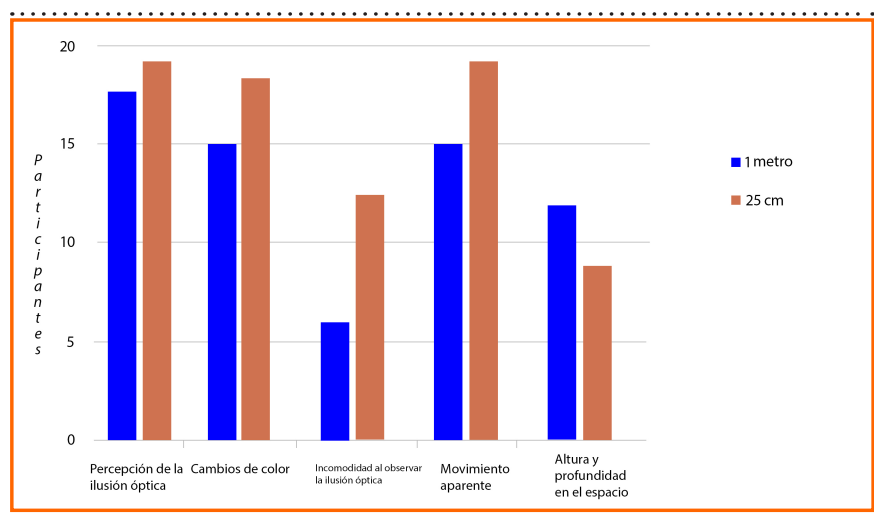

Tabla 3. Gráfico de resultados

En términos generales, las ilusiones ópticas dieron mejor resultado a 25 $\mathrm{cm}$, excepto a la hora de apreciar la distancia entre el contenido digital 


\section{artnodes}

https://artnodes.uoc.edu

y la pieza física, donde se producían más errores perceptivos a mayor distancia. Esto puede deberse a que, a mayor distancia respecto a la pieza, más difícil es hallar su posición correcta en el espacio.

La mayor diferencia en los resultados se encontraron en las molestias visuales ocasionadas al observar la pieza, siendo más acusada a $25 \mathrm{~cm}$ de distancia. En este caso, una mejor visualización del contenido digital y, por lo tanto, una mejor recepción del engaño provocaba una molestia mayor.

Otros motivos que pueden influir en la percepción de las ilusiones ópticas en RA son la resolución, el contraste o el rango de luminancia de las pantallas y la calidad de los dispositivos utilizados para visualizar la experiencia. En ocasiones se generaron efectos centelleantes de parpadeo debido a la reproducción digital de la imagen por parte del smartphone. También la aplicación Artivive tuvo influencia, ya que, en ciertas ocasiones, se generaba un traqueteo o vibración en la imagen que afectaba la percepción del efecto.

En investigaciones anteriores, uno de los problemas sugeridos en los diseños de RA es la percepción correcta de los objetos aumentados, siendo algunos de los problemas más comunes los causados por la profundidad y la iluminación (Gruber, Kalkofen, y Schmalstieg, 2010). En este caso, la visualización perceptualmente correcta a través de ilusiones ópticas puede ofrecer un nuevo campo de investigación a través del cual profundizar más sobre los problemas de percepción en RA. Sin embargo, todavía falta evidencia empírica de estas relaciones.

En la primera pieza, Modelo basado en la figura de Rotsnake, de Akiyoshi Kitaoka, la sensación de mareo o fatiga visual disminuyó ligeramente en la experiencia RA, gracias a que el efecto fue menos notorio 0 incluso nulo en algún caso. Varios participantes tuvieron la sensación de que las ruedas dejaron de girar en algún momento de la visualización, lo que permitió observar por más tiempo la ilusión. Este puede ser un punto de partida para la investigación sobre cómo se perciben las ilusiones ópticas a través de RA y cómo se ven afectadas por los dispositivos de visualización.

En la segunda pieza artística: Percepción errónea del color por influencia contaminante de un tercero, el color fue influenciado por el dispositivo utilizado. En algunos casos, los participantes informaron haber visto colores que no estaban representados en la pieza, como violeta, azul verdoso o rojo violáceo. En este caso, la experiencia está muy influenciada por la capacidad de resolución del dispositivo. Esto da como resultado señales de captación de colores en conflicto.

En la tercera pieza artística: 'Metamorfosis'. La distorsión anamórfica a través de la $R A$, el hecho de ver una serie de elementos indefinidos a diferentes distancias de profundidad, dificultaba la colocación de las piezas para, aproximadamente, la mitad de los participantes. Esto sugiere que la posición de elementos a diferentes profundidades puede generar problemas de percepción de la distancia en RA. La forma mal definida de los elementos RA puede enfatizar los problemas de percepción de profundidad. Este puede ser un campo
Arte y realidad aumentada. llusiones ópticas en el espacio híbrido

de interés a través del cual investigar sobre juicios de profundidad y diseño espacial en RA.

\section{Conclusión}

En este artículo se estudian las ilusiones ópticas en piezas artísticas creadas mediante RA, comparando la interacción de objetos virtuales con el espacio real. Para la parte experimental se utilizan, a modo de modelos, tres ilustraciones creadas por el autor que funcionan como marcador sobre el que se superponen una serie de elementos virtuales que generan ilusiones ópticas.

Para la creación de aplicaciones de RA se utiliza la herramienta Artivive. El diseño del contenido digital y su posterior inclusión en dicha aplicación resultó la opción más apropiada en comparación con otros medios para crear realidad aumentada, por su intuitiva interfaz, especialmente pensada para artistas e ilustradores, y por los buenos resultados al detectar y superponer las capas de contenido virtual sobre el espacio físico. Los participantes debían visualizar las piezas a través de dicha aplicación para poder ver los elementos virtuales, colocados a diferentes distancias.

Los resultados obtenidos en el estudio revelan que, a pesar de que las ilusiones ópticas examinadas continúan ocurriendo, la participación de más elementos involucrados en su materialización, como los dispositivos de visualización (teléfonos inteligentes) provoca alteraciones en estas. Por lo tanto, la percepción correcta del objeto aumentado sigue siendo un desafío esencial (Merino, Bergel, y Nierstrasz, 2018). En algunos momentos, al realizar la experiencia de RA, se observaron vibraciones que se confundieron con el efecto generado por las ilusiones ópticas, esto fue provocado por problemas de software, lo que demuestra que existe un amplio margen de mejora basado en el progreso tecnológico de los dispositivos y abre una posible vía de investigación sobre cómo se ven afectadas las ilusiones ópticas a través de su visualización en teléfonos móviles. En cuanto a la ilusión de percepción errónea del color, fue inesperado que el uso de la RA enfatizara la ilusión de terceros colores a partir de los presentes en la pieza. El hecho de que el color pierda intensidad cuando se ve a través de la RA se debe a las limitaciones de los dispositivos móviles. Esto abre posibles vías de investigación para mejorar la captura de color en aplicaciones de RA a través de ilusiones ópticas basadas en la percepción errónea del color a través de la influencia contaminante de un tercero.

Este estudio tiene como objetivo sentar las bases para mejorar la comprensión de la percepción de ilusiones ópticas en RA a través de la práctica artística y ser útil para futuras investigaciones que busquen profundizar en aspectos relacionados con este fenómeno. Además, el uso cada vez más extendido de la RA sugiere continuar desarrollando investigaciones en torno al tema de las ilusiones ópticas en entornos de RA. 


\section{Referencias bibliográficas}

Ardelean, Sergiu, y Codin Popescu. 2017. Artivive. Viena. https://artivive. com/about/.

Bach, Michael, y Charlotte M. Poloschek. 2006. «0ptical Illusions». ACNRAdvances in Clinical Neuroscience \& Rehabilitation, 6 n.o 2.

Chamberlain, Rebecca, Jennifer Drake, Aaron Kozbelt, Rachel Hickman, Joseph Siev, y Johan Wagemans. 2017. «Artists as Experts in Visual Cognition: An Update». Psychology of Aesthetics Creativity and the Arts, 13 n.0 1. D0I: https://doi.org/10.1037/aca0000156.

Conway, Bevil R., Akiyoshi Kitaoka, Arash Yazdanbakhsh, Christopher C. Pack, y Margaret S. Livingstone, 2005. «Neural Basis for a Powerful Static Motion Illusion». Journal of Neuroscience, 8 June, 2005, 25 n.o 23: 5651-5656. D0I: https://doi.org/10.1523/ JNEUROSCI.1084-05.2005.

Coren, Stanley, y Joan Girgus. 2020. Seeing is Deceiving: The Psychology of Visual IIlusions. DOI: https://doi.org/10.4324/9781003050681.

Cretenoud, Aline F., Harun Karimpur, Lukasz Grzeczkowski, Gregory Francis, Kai Hamburger, y Michael H. Herzog. 2019. «Factors underlying visual illusions are illusion-specific but not feature-specific». Journal of Vision 19 n.o 14:12. D0l: https://doi.org/10.1167/19.14.12.

Cuevas, María. 2010. Distorsión, equívocos y ambigüedades: las ilusiones ópticas en el arte. Madrid: Universidad Complutense de Madrid. Biblioteca Histórica. UCM, Facultad de Bellas Artes.

Díaz Padilla, Ramón. 2010. Distorsión, equívocos y ambigüedades. las ilusiones ópticas en el arte. Madrid: Universidad Complutense de Madrid. Biblioteca Histórica. UCM, Facultad de Bellas Artes.

Dodgson, Neil. 2008. «Regularity and Randomness in Bridget Riley's Aarly 0p Art». En Computational Aesthetics in Graphics, Visualization, and Imaging, 107-114, editado por Douglas W. Cunningham, Victoria Interrante, Paul Brown, y Jon McCormack. The Eurographics Association. DOI: https://doi.org/10.2312/COMPAESTH/ COMPAESTH08/107-114.

Gomez-Villa, Alexander, Adrian Martín, Javier Vazquez-Corral, Jesús Malo, y Marcelo Bertalmío. 2019. «Synthesizing Visual Illusions Using Generative Adversarial Networks». En Computer Vision and Pattern Recognition (cs.CV). Nueva York: Cornell University.

Gruber, Lukas, Denis Kalkofen, y Dieter Schmalstieg. 2010. «Color harmonization for Augmented Reality». 2010 IEEE International Symposium on Mixed and Augmented Reality, 13-16 octubre 2010. Seúl. htttps://10.1109/ISMAR.2010.5643580.

Hibbard, Paul B., Alice E. Haines, y Rebecca L. Hornsey, 2017. «Magnitude, precision, and realism of depth perception in stereoscopic vision». Cognitive Research: Principles and Implications, 2. D0I: https://doi.org/10.1186/s41235-017-0062-7.

Huang, Huize. 2020 «Parsing Creative Process in 0p Art Through Code-Driven Drawing Tools: Alternating Digital Art and Generative Art Practices». Tesis. Claremont, California: Claremont College. https://scholarship.claremont.edu/scripps_theses/1483.
Lin, Chiuhsiang Joe, Dino Caesaron, y Bereket Haile Woldegiorgis. 2019. «The Effects of Augmented Reality Interaction Techniques on Egocentric Distance Estimation Accuracy». Applied Sciences, 9 n.o 21. DOI: https://doi.org/10.3390/app9214652.

Merino, Leonel, Alexandre Bergel, y Oscar Nierstrasz. 2018. «0vercoming Issues of 3D Software Visualization through Immersive Augmented Reality». IEEE Working Conference on Software Visualization (VISSOFT 2018). Madrid. DOl: https://doi.org/10.1109/ VISSOFT.2018.00014.

Nather, Francisco Carlos, Fernando Figueiredo Mecca, y José Lino Bueno. 2012. "Motion illusions caused by paintings of Op Art distort the perception of time». Department of Psychology, University of São Paulo, Faculty of Philosophy Sciences and Letters of Ribeirão Preto, São Paulo, Brazil.

Oliver, Julian. 2007. «Level Head». Berlín. https://www.digitalartarchive.at/database/general/work/level-head.html. Acceso 14 septiembre, 2020. Website: https://julianoliver.com/levelhead/.

Pappenheimer, Will. 2016. «Transitional Spaces». Nueva York, EE.UU. http://www.willpap-projects.com/Transitional_Spaces/ Transitional_Spaces.html.

Peillard, Etienne, Ferran Argelaguet, Jean-Marie Normand, Anatole Lécuyer, y Guillaume Moreau. 2019. «Studying Exocentric Distance Perception in Optical See-Through Augmented Reality». IEEE International Symposium on Mixed and Augmented Reality (ISMAR), Beijing, China, 2019. DOI: https://doi.org/10.1109/ISMAR.2019.00-13.

Penrose, Lionel S., y Roger Penrose. 1958. «Impossible objects: A special type of visual illusion». British Journal of Psychology, 49: 31-33. DOl: https://doi.org/10.1111/j.2044-8295.1958.tb00634.x.

Rhodes, Geoffrey Alan. 2018. «Augmented Reality in Art: Aesthetics and Material for Expression». En Augmented Reality Art. Springer Series on Cultural Computing, editado por Geroimenko V. Springer, Cham. DOI: https://doi.org/10.1007/978-3-319-69932-5_7.

Rycroft, Simon. 2005. «The Nature of Op Art: Bridget Riley and the Art of Nonrepresentation». Environment and Planning D: Society and Space, 23 n.o 3:351-371. https://doi.org/10.1068/d54j.

Singh, Gurjot, Stepehn R. Ellis, y J. Edward Swan. 2020. «The Effect of Focal Distance, Age, and Brightness on Near-Field Augmented Reality Depth Matching». En IEEE Transactions on Visualization and Computer Graphics, 26 n.o 2: 1385-1398., DOl: https://doi. org/10.1109/TVCG.2018.2869729.

Slater, Mel, Cristina Gonzalez-Liencres, Patrick Haggard, Charlotte Vinkers, Rebecca Gregory-Clarke, Steve Jelley, Zillah Watson, Graham Breen, Raz Schwarz, William Steptoe, Dalila Szostak, Shivashankar Halan, Deborah Fox, y Jeremy Silver. 2020. «The Ethics of Realism in Virtual and Augmented Reality». Front. Virtual Real., 3 marzo, 2020. DOI: https://doi.org/10.3389/frvir.2020.00001.

Smith, Dunaway. 2020. «AR Stardust». https://www.dunawaysmith. com/site/2020/01/27/stardustar-art-illustration/ 2020. Website: www.dunawaysmith.com. 


\section{artnodes}

https://artnodes.uoc.edu

Thiel, Tamiko. 2018. «Biography». Munich. http://mission-base.com/ tamiko/cv.html\#Bio. Acceso 26 septiembre, 2020. Website: www. tamikothiel.com.

Thyssen-Bornemisza National Museum. 2018. «Victor Vasarely. El nacimiento del 0p Art». https://www.museothyssen.org/exposiciones/victor-vasarely-nacimiento-op-art. Acceso 12 octubre 2020.

Wade, Nicholas J. 2011. «Eye contricks». I-Perception, 2 n.o 5: 486501. D0l: https://doi.org/10.1068/i0442.
Arte y realidad aumentada. llusiones ópticas en el espacio híbrido

Wade, Nicholas J. 1978. «0p Art and Visual Perception». Perception, 7 n.0 1: pp. 21-46. DOl: https://doi.org/10.1068/p070021.

Zanker, Johannes, y Robin Walker. 2004. «A new look at Op art: Towards a simple explanation of illusory motion". Die Naturwissenschaften, 91 n.o 4: 149-156. DOI: https://doi.org/10.1007/ s00114-004-0511-2.

Zeki, Semir, y M. Lamb. 1994. «The neurology of kinetic art». Brain: a Journal of Neurology, 117, n.o 3: 607-636. D0l: https://doi. org/10.1093/brain/117.3.607. 


\section{artnodes}

https://artnodes.uoc.edu

Arte y realidad aumentada. Ilusiones ópticas en el espacio híbrido

\section{CV}

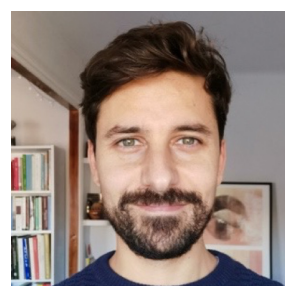

\section{Borja Jaume Pérez}

Universidad Complutense de Madrid

borjaume@ucm.es

Borjaume es un artista digital que trabaja en los campos del dibujo experimental, el arte interactivo y la realidad virtual. Doctorando de la Facultad de Bellas Artes por la Universidad Complutense de Madrid, se ha especializado en dibujo y realidad virtual. Su práctica ha evolucionado hacia experiencias inmersivas a través de la mezcla de técnicas tradicionales y digitales, expandiéndose más allá de la superficie bidimensional para crear dibujos híbridos en los que habla sobre el conflicto cotidiano entre los dispositivos digitales y analógicos, a la par que establece un diálogo entre el cuerpo y el espacio. Ha realizado talleres sobre realidad aumentada con Unity, talleres sobre dibujo digital, estudio de volumen y de forma a través de programas de libre acceso como Krita o Inkscape, y ha participado como ponente en varios congresos, analizando las posibilidades artísticas que ofrece el uso de herramientas de realidad extendida y de software libre como Arduino, Processing o Pure Data. Ha participado en el programa internacional IMACLA fruto de la colaboración entre la Facultad de Bellas Artes UCM y la Oslo National Academy of the Arts (Khio), tratando las posibilidades del dibujo interactivo a través de realidad virtual. 